$\underline{\text { Preprint typeset in JHEP style - HYPER VERSION }}$

\title{
The covariant entropy bound in gravitational collapse
}

\author{
Sijie Gao and José P. S. Lemos \\ Centro Multidisciplinar de Astrofísica - CENTRA \\ Departamento de Física, Instituto Superior Técnico \\ Av. Rovisco Pais 1, 1049-001 Lisboa, Portugal \\ E-mail: sijie@fisica.ist.utl.pt, lemos@fisica.ist.utl.pt
}

\begin{abstract}
We study the covariant entropy bound in the context of gravitational collapse. First, we discuss critically the heuristic arguments advanced by Bousso. Then we solve the problem through an exact model: a Tolman-Bondi dust shell collapsing into a Schwarzschild black hole. After the collapse, a new black hole with a larger mass is formed. The horizon, $L$, of the old black hole then terminates at the singularity. We show that the entropy crossing $L$ does not exceed a quarter of the area of the old horizon. Therefore, the covariant entropy bound is satisfied in this process.
\end{abstract}

KEYwords: Space-Time Symmetries, Black Holes. 


\section{Contents}

1. Introduction

2. A thick shell collapsing into a black hole

3. Testing the covariant entropy bound 7

4. Discussions and conclusions 10

\section{Introduction}

Bousso's covariant entropy conjecture says [1]: let $A$ be the area of a connected twodimensional spatial surface $B$ contained in a four-dimensional spacetime $\mathcal{M}$. Let $L$ be a hypersurface bounded by $B$ and generated by surface orthogonal null geodesics with nonpositive expansion. Then the total entropy, $S$, contained on $L$ can not exceed one quarter of the area, i.e., $S \leq A / 4$.

Evidences supporting this conjecture have been found in cosmological solutions and other matter systems [1]-[5]. One example discussed by Bousso [1] is a shell collapsing into a black hole. We now briefly review the arguments given in [1]. Consider a shell with mass $M$, collapsing into a black hole with original mass $M_{0}$. The apparent horizon $L$ is located at $r=r_{0}$ before the collapse. Once the shell has crossed the light-sheet $L$ at $r_{0}$, the null generators of $L$ will be moving in a Schwarzschild interior of mass $\tilde{M}=M+r_{0} / 2$ and hit the singularity $r=0$ eventually. Since only the entropy crossing $L$ is relevant in the entropy bound, we consider the case where the outer surface of the shell meets $L$ right at the singularity (See figure 1).

The author in [1] claims that the meeting occurs at proper time

$$
\Delta \tau_{\text {dead }}=r_{0}+2 \tilde{M} \ln \left(1-\frac{r_{0}}{2 \tilde{M}}\right) \approx \frac{r_{0}^{2}}{4 \tilde{M}}
$$

The approximation above was made under the condition $r_{0} \ll 2 \tilde{M}$ or $r_{0} \ll 2 M$. The maximum proper thickness of the shell is then approximated as

$$
w_{\max } \approx \frac{r_{0}^{2}}{2 \tilde{M}} .
$$

Next, the author used the Bekenstein's bound to estimate the maximum entropy of shell and obtained

$$
S=2 \pi w M .
$$




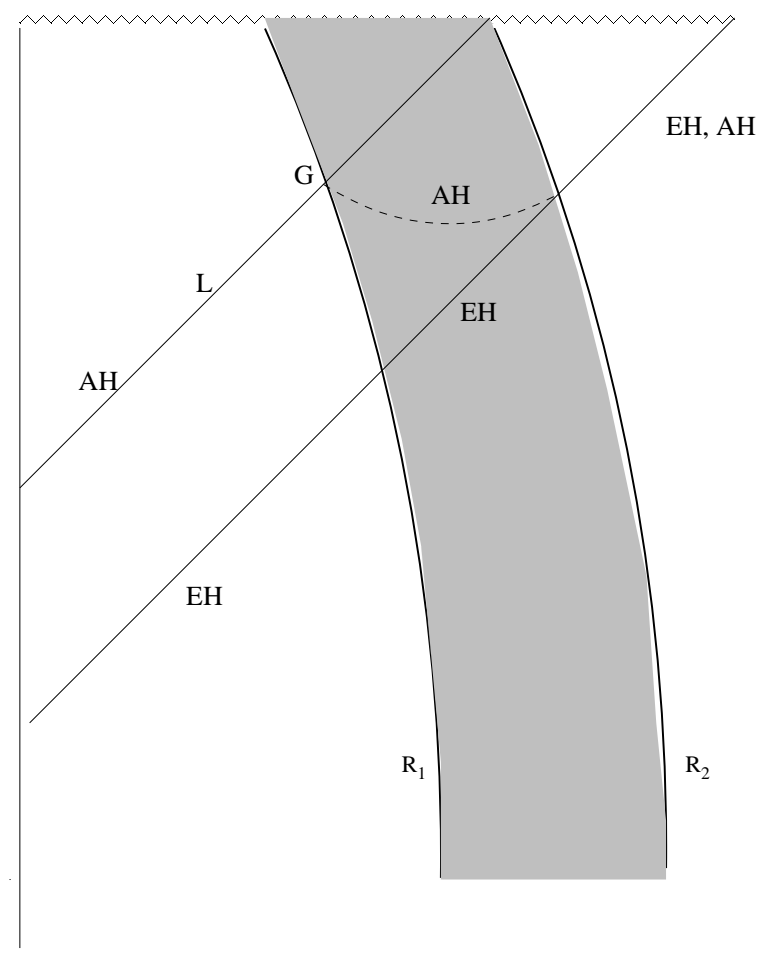

Figure 1: A Tolman-Bondi dust shell falling into a black hole. AH represents the apparent horizon and EH represents the event horizon.

The condition $w \leq w_{\max }$ gives

$$
S_{\max }=\pi r_{0}^{2} \frac{M}{\tilde{M}} \leq \frac{A}{4}
$$

The expression above shows that the covariant entropy bound is preserved. We see that the maximum thickness of the shell plays an essential role in deriving the bound. However, the author did not show explicitly how $\Delta \tau_{\text {dead }}$ was derived and did not explain clearly its physical meaning. We shall show that the right-hand side of eq. (1.1) can only be interpreted as a coordinate distance. The Schwarzschild metric takes the form

$$
d s^{2}=-\left(1-\frac{2 \tilde{M}}{r}\right) d t^{2}+\left(1-\frac{2 \tilde{M}}{r}\right)^{-1} d r^{2}+r^{2} d \Omega^{2} .
$$

Inside the black hole the coordinate $t$ plays the role of distance, while $r$ plays the role of time. For a null geodesic starting at $r=r_{0}<2 M$ and ending up at the singularity $r=0$, the difference in $t$ is

$$
\begin{aligned}
\Delta t & =\int_{0}^{r_{0}} \frac{r}{r-2 \tilde{M}} d r \\
& =r_{0}+2 \tilde{M} \ln \left(1-\frac{r_{0}}{2 \tilde{M}}\right) \approx \frac{r_{0}^{2}}{4 \tilde{M}},
\end{aligned}
$$

which is just the right-side of eq. (1.1). From our calculation above, the interpretation of $\Delta t$ is clear: inside the black hole, $r<2 \tilde{M}$, there are comoving observers associated with 
coordinates $(t, r)$ with $-(\partial / \partial r)^{a}$ tangent to the world lines of the observers. Different from the outside of the black hole, these observers are no longer stationary. Suppose that we choose an observer $A$ and at the moment $r=r_{0}$, an outgoing light signal is sent from this observer in the radial direction and reach another observer $B$ at the singularity $r=0$. Then, $\Delta t$ is the coordinate distance between $A$ and $B$. The observer $A$ corresponds to the middle of the collapsing shell and $B$ to the outer surface. According to [1], the finite null geodesic will restrict the thickness of the shell. Eq. (1.1) is used in [1] to estimate the upper bound of the proper thickness of the shell given in eq. (1.2). However, the proper distance, $\Delta \tau$, between two comoving observers at the moment $r_{0}$ differs from the coordinate distance by

$$
\Delta \tau=\Delta t \sqrt{\frac{2 \tilde{M}}{r_{0}}-1} .
$$

For $r_{0} \ll 2 \tilde{M}$, we have $\Delta \tau \gg \Delta t$. This means that the maximum width of the shell can be much larger than what is given in [1]. Now, we shall argue furthermore that the thickness of the shell may be arbitrarily large, i.e., the idea that a finite light ray gives rise to an upper bound of proper distance between observers passing through it is simply wrong. The above maximum thickness is obtained by assuming that the shell moves at the moment $r=r_{0}$ with the comoving observers inside the black hole. There is no explanation in [1] why this particular motion is preferred. Now we show the proper length can be arbitrarily large if motions of the shell can be arbitrarily chosen. The argument can be easily illustrated in the case of special relativity.

In figure 2, $L$ represents a light sheet in Minkowski spacetime. $\mathrm{S}$ and $\mathrm{S}$ ' are two inertial reference frames with coordinates $(t, x)$ and $\left(t^{\prime}, x^{\prime}\right)$, respectively. Let $v$ be the relative velocity between $\mathrm{S}$ and $\mathrm{S}$ '. Then the Lorentz transformation is given by the two equations:

$$
\begin{aligned}
t^{\prime} & =\gamma(t-v x), \\
x^{\prime} & =\gamma(x-v t) .
\end{aligned}
$$

Figure 2 corresponds to a negative $v$. The proper length between the two observers in $\mathrm{S}$ passing through $L$ is $|O C| \equiv L_{0}$. Here $L_{0}$ is analogous to the proper length of the shell which follows the comoving observers inside the black hole. It is not difficult to find that the coordinates of $B$ in $\mathrm{S}$ are

$$
\begin{aligned}
t_{B} & =\frac{v}{1+v} L_{0}, \\
x_{B} & =\frac{L_{0}}{1+v} .
\end{aligned}
$$

Then the proper length of $O B$ is easily obtained from the Minkowski metric

$$
|O B|=L_{0} \sqrt{\frac{1-v}{1+v}} .
$$

Therefore, when $v$ is arbitrarily close to $-1,|O B|$ will be arbitrarily large, i.e., the finite light sheet $L$ can not put an upper bound on the proper length between observers passing it. 


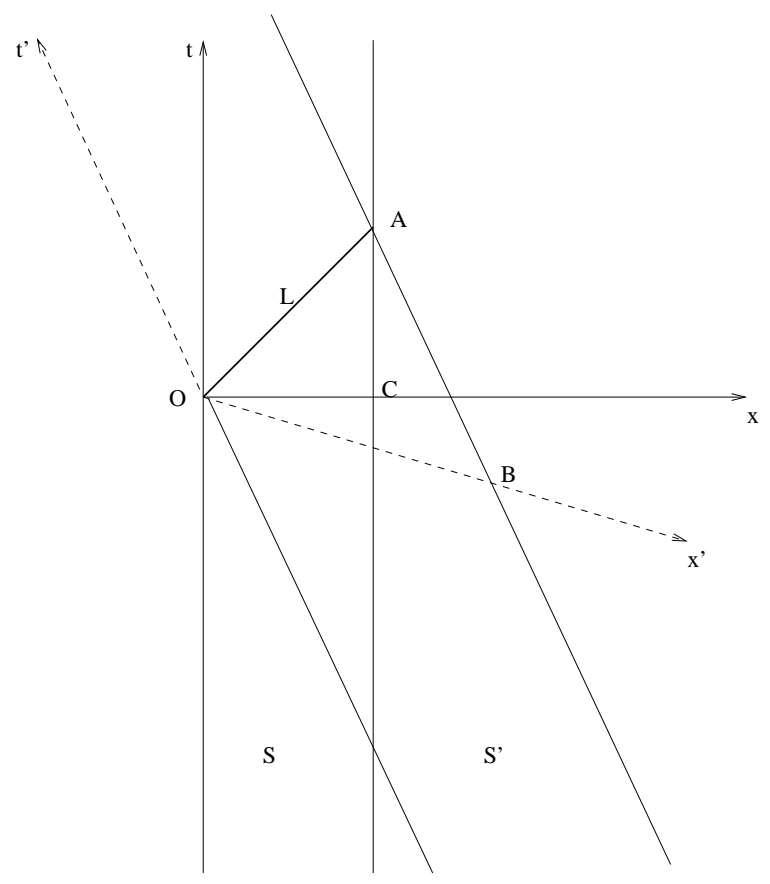

Figure 2: A spacetime diagram for observers in references $S$ and $S^{\prime}$ passing through the light-sheet $L$ in Minkowski spacetime, respectively. The proper distances between the two observers in $S$ and $S^{\prime}$ are $|O C|$ and $|O B|$, respectively.

One can generalize the arguments above with some ease to a curved background. So the estimation of the maximum width of the shell given in [1] is not valid. Another problem in Bousso's analysis is that the shell of finite thickness is simplified by an infinitely thin shell. So the details of the shell become irrelevant. It is unclear to us whether this approximation is valid or accurate.

To clarify these issues, we first construct an exact solution of a shell filled with TolmanBondi dust collapsing into a black hole. The regions inside the inner boundary and outside the outer boundary of the shell are described by Schwarzschild metrics with different masses. Following the discussion on the homogeneous cosmology in [1], we assume that the entropy of the shell is invariant in a comoving volume. We then test the bound for $L$ during the collapse. Our results show that there is no violation for the covariant entropy bound in the classical regime.

\section{A thick shell collapsing into a black hole}

Relativistic thin shell solutions have been studied by many authors (see e.g., 6]-9]). Thin shells are considered as zero thickness objects. Consequently, the extrinsic curvature across the shell becomes discontinuous. The jump of the extrinsic curvature is related to the surface stress-energy tensor. A spherical thick shell solution was found and corrections to the thin shell dynamics were discussed by Khakshournia and Mansouri [10]. The shell is immersed in two different spherically symmetric space-times. In contrast to thin shell cases, the junction conditions for thick shells then become that the extrinsic curvatures 
across the inner boundary and the outer boundary of the shell are continuous, respectively. We apply these conditions to a spacetime where a spherical thick shell is immersed in two Schwarzschild spacetimes. In this solution, a Schwarzschild black hole with mass $M_{0}=r_{0} / 2$ is already there. Then a shell with gravitational mass $M$ collapses into it and forms a new black hole with mass $\tilde{M}=M_{0}+M$. The shell consists of Tolman-Bondi dust. One assigns to each layer of the thick shell a comoving coordinate $R$ with an energy per unit mass $E(R)$. We will only discuss the marginally bound case with $E(R)=0$, which is described by the metric 11]

$$
d s^{2}=-d T^{2}+r^{\prime 2} d R^{2}+r^{2}(T, R)\left(d \theta^{2}+\sin ^{2} \theta d \varphi^{2}\right),
$$

where $R_{1} \leq R \leq R_{2}$ and $r^{\prime}=\partial r / \partial R$. We denote by $\Sigma_{1}$ and $\Sigma_{2}$ the inner boundary $R=R_{1}$ and the outer boundary $R=R_{2}$, respectively. The function $r(T, R)$ is the solution of

$$
\frac{1}{2}\left(\frac{\partial r}{\partial T}\right)^{2}=\frac{M(R)}{r}
$$

where $M(R)$ is the effective gravitational mass within $R$. The self-similar solution in this case is 11]

$$
\begin{aligned}
M(R) & =R \\
r(T, R) & =\left(\frac{9}{2} R\right)^{1 / 3}(B R-T)^{2 / 3},
\end{aligned}
$$

where the constant $B$ is a homogeneity parameter. The shell divides the spacetime into three regions. Denote the region inside $\Sigma_{1}$ by $\mathcal{M}_{\text {in }}$, outside $\Sigma_{2}$ by $\mathcal{M}_{\text {out }}$ and the shell by $\mathcal{M}_{\mathrm{s}} \cdot \mathcal{M}_{\text {in }}$ is described by the Schwarzschild metric:

$$
d s^{2}=-\left(1-\frac{2 M_{0}}{r}\right) d t^{2}+\left(1-\frac{2 M_{0}}{r}\right)^{-1} d r^{2}+r^{2} d \theta^{2}+r^{2} \sin ^{2} \theta d \varphi^{2},
$$

where $r<r\left(T, R_{1}\right) . \quad M_{0}$ needs to be determined by boundary conditions of $\Sigma_{1} \cdot \Sigma_{1}$ is a timelike hypersurface described by $R=R_{1}$. Following [10], we require the continuity of the extrinsic curvature tensor $K_{a b}$ of $\Sigma_{1}$. To calculate $K_{a b}$, consider the tangent vector of a comoving observer on $\Sigma_{1}$ :

$$
\left(\frac{\partial}{\partial T}\right)^{a}=\dot{t}\left(\frac{\partial}{\partial t}\right)^{a}+\dot{r}\left(\frac{\partial}{\partial r}\right)^{a}
$$

where $\dot{r}=\partial r\left(T, R_{1}\right) / \partial T$. $\dot{t}$ can be solved by the fact that $T$ is the proper time and therefore $g_{a b}(\partial / \partial T)^{a}(\partial / \partial T)^{b}=-1$ in $\mathcal{M}_{\mathbf{s}}$. The matching conditions require that $(\partial / \partial T)^{a}$ be also normalized when measured by the metric of $\mathcal{M}_{\text {in }}$. Hence, from eqs. (2.4) and (2.5), we have

$$
-\left(1-\frac{2 M_{0}}{r}\right) \dot{t}^{2}+\left(1-\frac{2 M_{0}}{r}\right)^{-1} \dot{r}^{2}=-1,
$$

and then

$$
\dot{t}=\left(1-\frac{2 M_{0}}{r}\right)^{-1 / 2} \sqrt{1+\left(1-\frac{2 M_{0}}{r}\right)^{-1} \dot{r}^{2}} .
$$


The unit tangent field orthogonal to $\Sigma_{1}$ in $\mathcal{M}_{\text {in }}$ can be calculated from eqs. (2.4) and (2.5) as

$$
n_{a}=-\dot{r}(d t)_{a}+\left(1-\frac{2 M_{0}}{r}\right)^{-1} \sqrt{1-\frac{2 M_{0}}{r}+\dot{r}^{2}}(d r)_{a} .
$$

The extrinsic curvature, $K_{a b}$, of $\Sigma_{1}$ is defined by [12]

$$
K_{a b}=\nabla_{a} n_{b}
$$

Straightforward calculation shows that the $\theta-\theta$ component of $K_{a b}$ is

$$
K_{\theta \theta}=r \sqrt{1-\frac{2 M_{0}}{r}+\dot{r}^{2}} .
$$

It is understood that all quantities are valuated on $\Sigma_{1}$. Similar calculation gives the $\theta-\theta$ component of $K_{a b}$ derived from the metric of $\mathcal{M}_{\mathrm{s}}$ :

$$
K_{\theta \theta}=r .
$$

By continuity, the right-hand sides of eqs. 2.10) and (2.11) must be equal. Then, from eqs. (2.2) and (2.3), we get immediately

$$
M_{0}=R_{1}
$$

This result is expected since $R_{1}$ would be the gravitational mass within $\Sigma_{1}$ if the region of $\mathcal{M}_{\text {in }}$ were filled with Tolman-Bondi dust described by eq. (2.1). Similarly, the spacetime exterior to the shell is Schwarzschild with mass $\tilde{M}=R_{2}$. In summary, the metric of the entire spacetime is

$$
d s^{2}= \begin{cases}-\left(1-\frac{2 M_{0}}{r}\right) d t^{2}+\left(1-\frac{2 M_{0}}{r}\right)^{-1} d r^{2}+r^{2} d \Omega^{2}, & \mathcal{M}_{\text {in }} \\ -d T^{2}+\frac{(3 B R-T)^{2}}{R^{4 / 3}(6 B R-6 T)^{2 / 3}} d R^{2}+\left(\frac{9}{2} R\right)^{2 / 3}(B R-T)^{4 / 3} d \Omega^{2}, & R_{1}<R<R_{2} \\ -\left(1-\frac{2 \tilde{M}}{r}\right) d t^{2}+\left(1-\frac{2 \tilde{M}}{r}\right)^{-1} d r^{2}+r^{2} d \Omega^{2}, & \mathcal{M}_{\text {out }}\end{cases}
$$

where $M_{0}=R_{1}$ and $\tilde{M}=R_{2}$. A future singularity is located at 11

$$
T=B R
$$

Since both $\mathcal{M}_{\text {in }}$ and $\mathcal{M}_{\text {out }}$ are Schwarzschild, the apparent horizon satisfies $r=2 M_{0}$ in $\mathcal{M}_{\text {in }}$ and $r=2 \tilde{M}$ in $\mathcal{M}_{\text {out }}$. To find out the apparent horizon inside the shell $\mathcal{M}_{\mathrm{s}}$, we calculate the expansion, $\theta$, of the outgoing null geodesics. The apparent horizon follows the equation $\theta=0$, which yields

$$
T=\left(-\frac{4}{3}+B\right) R, \quad R_{1} \leq R \leq R_{2}
$$


Now we show that this solution makes the apparent horizon in the spacetime continuous. ${ }^{1}$ Denote by $G$ the intersection of $L$ and $\Sigma_{1}$ (see figure 1). Note that $G$ is a 2-sphere. By continuity of spacetime, its proper area calculated from $\mathcal{M}_{\text {in }}$ side and $\mathcal{M}_{\mathrm{s}}$ side should be equal. Since the radii of 2 -spheres along $L$ in $\mathcal{M}_{\text {in }}$ are always $2 R_{1}$, we have immediately

$$
2 R_{1}=r\left(T, R_{1}\right)
$$

which gives

$$
T=\left(-\frac{4}{3}+B\right) R_{1}
$$

Similarly, we have the meeting time of the apparent horizon in $\mathcal{M}_{\text {out }}$ with $\Sigma_{2}$ :

$$
T=\left(-\frac{4}{3}+B\right) R_{2}
$$

Thus, the apparent horizon in $\mathcal{M}_{\mathrm{s}}$ determined by eq. 2.15) joins the other two portions of the apparent horizon in a continuous manner (see figure 1).

\section{Testing the covariant entropy bound}

In this section, we shall test the covariant entropy bound in the collapsing process. The light sheet is chosen to be $L$ with area $A=4 \pi\left(2 M_{0}\right)^{2}$. To specify the entropy of the shell, we generalize the prescription in [1] where entropies for cosmological models are defined. By applying the coordinate transformation

$$
\bar{R}=3 R^{1 / 3}
$$

to the metric (2.13) with $B=0$, we obtain the flat dust-filled Robertson-Walker universe:

$$
d s^{2}=-d T^{2}+a^{2}(T)\left(d \bar{R}^{2}+\bar{R}^{2} d \Omega^{2}\right),
$$

where $a(T)=\frac{1}{6^{1 / 3}} T^{2 / 3}$. According to [1, 2], the universe evolves adiabatically and consequently, the physical entropy density, $s(T)$, is diluted by cosmological expansion:

$$
s(T)=\frac{s_{0}}{a^{3}(T)} .
$$

The constant $s_{0}$ can be specified by assuming that the entropy may not exceed one per Plank volume at the Planck time $T_{P l}^{2}$ [泪]. Therefore, we choose $s$ such that

$$
s(T)=\frac{a^{3}\left(T_{P l}\right)}{a^{3}(T)} .
$$

\footnotetext{
${ }^{1}$ The apparent horizon was defined by Hawking 13 to be the outer boundary of the total trapped region in a spacelike slice. In that case, the apparent horizon moves discontinuously. In this paper, we adopt the generalized definition that the apparent horizon is the boundary of the total trapped region 14 (see also 12 ).

${ }^{2}$ In 迎, the Planck time is near a past singularity. In our case, a future singularity is encountered. Thus, we shall use time symmetry and impose a future boundary condition.
} 
To generalize the above treatment to the inhomogeneous case, we make the following modifications. During the inhomogeneous collapse, the physical entropy density $s$ is a function of both $T$ and $R$. We denote it by $s(T, R)$. In analogy to the dust universe, we assume that the shell evolves adiabatically. Thus, the entropy in a comoving volume is constant. From the metric (2.1), we have the volume, $d V$, in a thin shell between $R$ and $R+d R$ :

$$
d V=4 \pi r^{\prime}(T, R) r^{2}(T, R) d R .
$$

Thus

$$
s(T, R) d V=4 \pi s(T, R) r^{\prime}(T, R) r^{2}(T, R) d R
$$

should be independent of $T$ for fixed $d R$. Therefore,

$$
4 \pi s(T, R) r^{\prime}(T, R) r^{2}(T, R) \equiv S(R),
$$

where $S(R)$ is the entropy per unit length. We still require that the entropy density $s$ be smaller than one at the "Planck time". But the "Planck time" here is no longer equal to 1 in Planck units, as in the case of homogeneous cosmology. For the inhomogeneous shell, we need to redefine the "Planck time". From dimensional arguments, quantum gravitational effects become important when the radius of curvature becomes of the order of $l_{p}$ [15]. Therefore, we define that in an inhomogeneous collapse, the "Planck time" is the hypersurface $\mathcal{R}=1$, where $\mathcal{R}$ is the scalar curvature and its value is in Planck units. This generalized "Planck time" is consistent with the Planck time in homogeneous cases. For example, the scalar curvature of metric (3.2) is 1.33 at the Planck time and conversely, $\mathcal{R}=1$ corresponds to $T=1.15$. Solving $\mathcal{R}=1$ from the metric of the shell (2.13), we find

$$
T=T_{P l}(R)=\frac{1}{3}\left(6 B R-\sqrt{3} \sqrt{4+3 B^{2} R^{2}}\right) .
$$

Now we have the observer-dependent "Planck time" $T_{P l}(R)$. Analogously, we require that the physical entropy $s(T, R)$ be equal to 1 at $T=T_{P l}(R)$. Therefore, eq. (3.7) yields

$$
s(T, R)=\frac{r^{\prime}\left(T_{P l}(R), R\right) r^{2}\left(T_{P l}(R), R\right)}{r^{\prime}(T, R) r^{2}(T, R)} .
$$

Substituting eqs. (3.8) and (3.9) into eq. (3.7), we have the following simple result

$$
S(R)=8 \pi \text {. }
$$

Thus the total entropy enclosed in the shell is

$$
S=8 \pi\left(R_{2}-R_{1}\right)
$$

Now we are ready to test the covariant entropy bound. First, we shall choose the value of $M_{0}$. Then, we solve numerically for $R_{2}$ from the equation of the radial null geodesic which starts at $G$ and ends at the singularity. Once we have obtained $R_{2}$, the total entropy crossing $L$ can be obtained from eq. (3.11) immediately (remember that $R_{1}=M_{0}$ ). To check the bound, we define

$$
\text { Ratio } \equiv \frac{4 S}{A}
$$




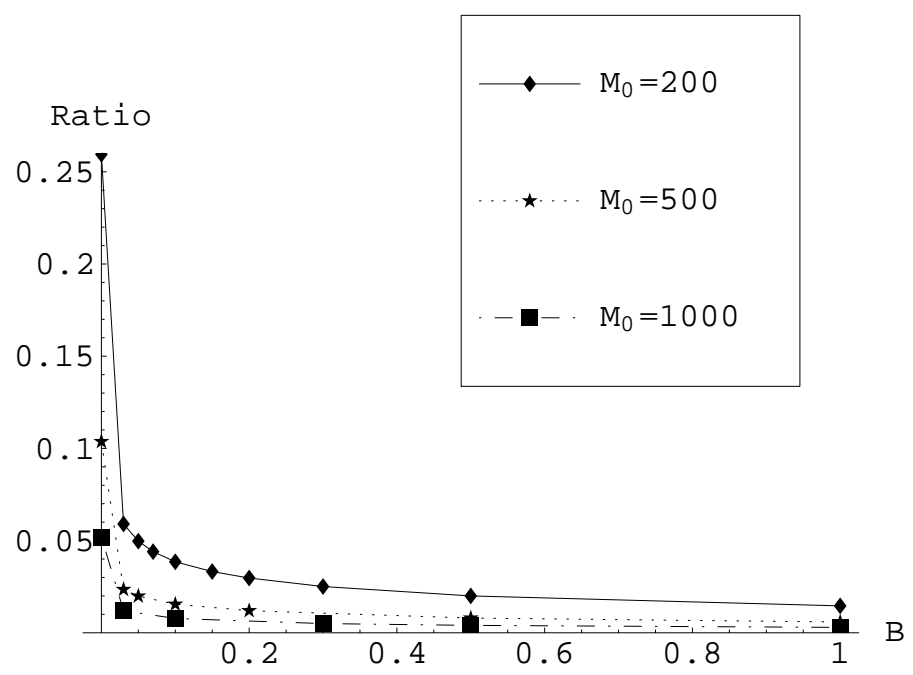

Figure 3: It is plotted the Ratio $\equiv 4 S / A$ as a function of the inhomogeneity parameter $B$, for three different large values of $M_{0}$. One sees that the entropy bound is satisfied for large values of $M_{0}$.

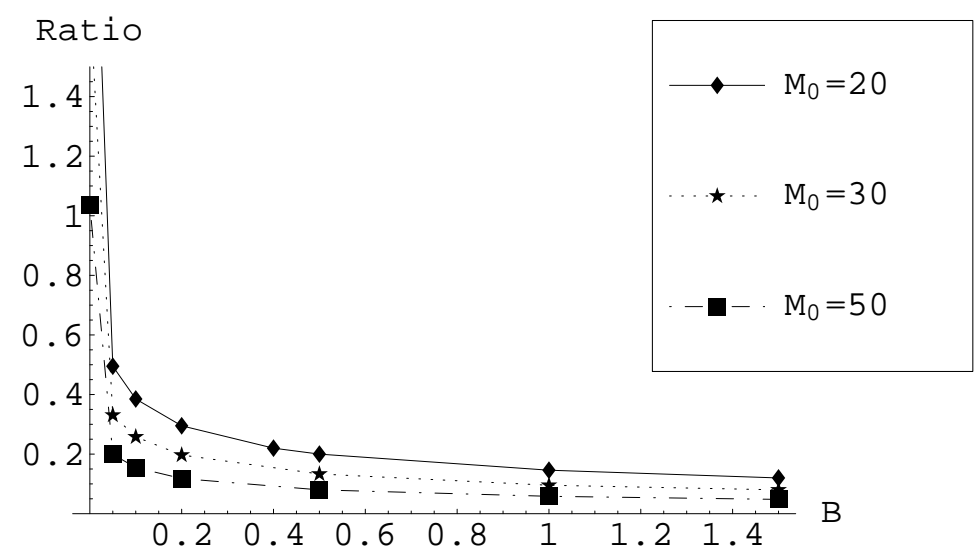

Figure 4: It is plotted the Ratio $\equiv 4 S / A$ as a function of the inhomogeneity parameter $B$, for three different small values of $M_{0}$. For $M_{0}<50$, violations occur at small values of $B$.

where $A=4 \pi\left(2 M_{0}\right)^{2}$ is the area of the light sheet. If Ratio $\leq 1$, the bound is satisfied. Otherwise, it is violated. Figure 3 shows that the bound holds for large values of $M_{0}$ and the Ratio decreases with $B$. However, figure 1 shows that violation occurs for $M_{0}<50$ and small values of $B$. Since $M_{0}=50$ is not near the Planck mass $m_{P l}=1$, it seems that the breakdown of classical relativity near the singularity cannot account for the violation. In order to explain this apparent violation, we consider the case of $B=0$, i.e., the flat dust-filled universe solution (3.2), because figure 1 indicates that the maximum violation occurs at $B=0$.

The solution (3.2) possesses a future crushing singularity at $T=0$. We choose a futuredirected light sheet $L$ starting from a 2 -sphere $B_{0}$ with coordinates $(T=-26.67, \bar{R}=$ 8.143). It is easy to check, from eq. (3.1), that this light sheet corresponds to that in the Tolman-Bondi spacetime when $M_{0}=20$ (well below the critical value $M_{0}=50$ ) and 


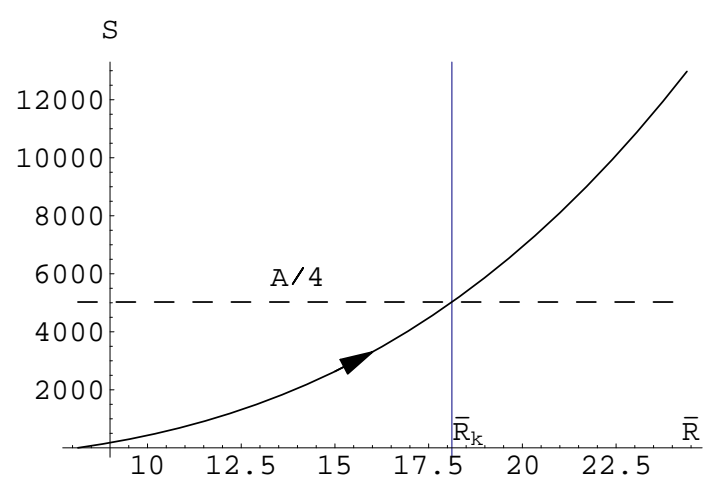

(a) The entropy crossing $L$ as a function of $\bar{R}$.

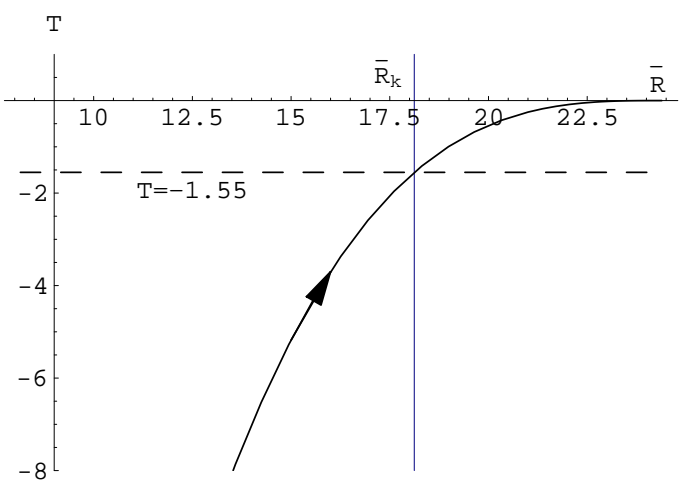

(b) A plot of the light sheet $L$ in $(T, \bar{R})$ coordinates.

Figure 5: The entropy $S$ exceeds $A / 4$ at $R>\bar{R}_{k}$. However, this region is within about a Planck time from the singularity, where a quantum description is necessary.

$B=0$. The 2 -sphere is obviously not very close to the singularity compared with the Planck scale. At $B_{0}$, the scalar curvature $\mathcal{R} \approx 0.00187$, which also indicates that classical description is sufficient in the neighborhood of $B_{0}$. However, as we shall now show, the violation of the bound is due to the entropy crossing $L$ within about one Planck time from the singularity, where quantum description is necessary. Indeed, figure $5 a$ illustrates the entropy distribution along $L$. The entropy saturates the $A / 4$ bound at $R=\bar{R}_{k} \approx 18.12$. If we continue to count the entropy after $\bar{R}_{k}$ all the way to the singularity, the bound will be exceeded by a wide margin. However, figure $5 b$ shows that $R=\bar{R}_{k}$ on the light-sheet corresponds to $T=-1.55$, which is around the Planck time. Since the portion of the shell crossing $L$ around this moment is in the Planck regime where quantum gravity description is required, it is reasonable to exclude the classical entropic reasonings in this region. As the entropy crossing the light-sheet within about a Planck length from the singularity should be excluded, the entropy bound still holds.

\section{Discussions and conclusions}

We have constructed an exact solution which corresponds to a Tolman-Bondi dust shell collapsing into a Schwarzschild black hole with mass $M_{0}$. The shell just crosses entirely the light-sheet $L$ which is terminated at the singularity. In the spirit of Bousso's assumptions, the entropy in a comoving volume of the shell is assumed to be a constant. We also set a future boundary condition, in contrast to Bousso's past boundary condition, to specify the entropy. It is always more difficult to argue on plausible final boundary conditions than on initial ones. However, time symmetry (an issue far from being settled) can be invoked to impose time symmetric boundary conditions. Our numerical results show that for $M_{0} \gtrsim 50$, the entropy of the shell does not exceed one quarter of the area of the black hole, while for $M_{0} \lesssim 50$, the entropy exceeds one quarter of the area. The later case appears to violate the covariant entropy bound. However, this violation can be interpreted as over-counting the entropy residing in the 
Planck regime (very close to the singularity). This part of entropy should not be included because classical relativity breaks down in that region. Therefore, we conclude that there is no violation on the covariant entropy bound during the gravitational collapse.

\section{Acknowledgments}

This work was partially funded by Fundação para a Ciência e Tecnologia (FCT) — Portugal through project SAPIENS 36280/99. SG acknowledges financial support from FCT grant SFRH/BPD/10078/2002. JPSL thanks Observatório Nacional do Rio de Janeiro for hospitality.

\section{References}

[1] R. Bousso, A covariant entropy conjecture, J. High Energy Phys. 07 (1999) 004 hep-th/9905177.

[2] R. Bousso, The holographic principle, Rev. Mod. Phys. 74 (2002) 825 hep-th/0203101.

[3] V. Husain, Probing entropy bounds with scalar field spacetimes, Phys. Rev. D 69 (2004) 084002 gr-qc/0307070.

[4] E.E. Flanagan, D. Marolf and R.M. Wald, Proof of classical versions of the bousso entropy bound and of the generalized second law, Phys. Rev. D 62 (2000) 084035 hep-th/9908070.

[5] R. Bousso, E.E. Flanagan and D. Marolf, Simple sufficient conditions for the generalized covariant entropy bound, Phys. Rev. D 68 (2003) 064001 hep-th/0305149].

[6] W. Israel, Singular hypersurfaces and thin shells in general relativity, Nuovo Cim. 44B (1966) 1, erratum ibid. 48B (1967) 463.

[7] P.R. Brady, J. Louko and E. Poisson, Stability of a shell around a black hole, Phys. Rev. D 44 (1991) 1891.

[8] J. Frauendiener, C. Hoenselaers and W. Konard, A shell around a black hole, Class. and Quant. Grav. 7 (1990) 585.

[9] S.M. C.V. Goncalves, Relativistic shells: dynamics, horizons and shell crossing, Phys. Rev. D 66 (2002) 084021 gr-qc/0212124.

[10] S. Khakshournia and R. Mansouri,Dynamics of general relativistic spherically symmetric dust thick shells, Gen. Rel. Grav. 34 (2004) 1847.

[11] J.P.S. Lemos, On naked singularities in selfsimilar Tolman-Bondi space-times, Phys. Lett. A 158 (1991) 279.

[12] R.M. Wald, General relativity, University of Chicago Press, Chicago 1984.

[13] S.W. Hawking, The event horizon, in Black holes, C. DeWitt and B.S. DeWitt eds., Gordon and Breach Science Publishers, New York 1972.

[14] D.M. Eardley and L. Smarr, Time function in numerical relativity. Marginally bound dust collapse, Phys. Rev. D 19 (1979) 2239.

[15] S.W. Hawking and G.F.R. Ellis, The large scale structure of space-time, Cambridge University Press, Cambridge 1973. 